\title{
Assessment of Biofilm Formation and Resistance to Imipenem and Ciprofloxacin among Clinical Isolates of Acinetobacter baumannii in Tehran
}

\author{
Ahya Abdi-Ali ${ }^{1, *}$; Saghar Hendiani ${ }^{1}$; Parisa Mohammadi ${ }^{1}$; Sara Gharavi ${ }^{1}$ \\ ${ }^{1}$ Department of Biology, Faculty of Science, Alzahra University, Tehran, IR Iran \\ *Corresponding author: Ahya Abdi-Ali, Department of Biology, Faculty of Science, Alzahra University, Tehran, IR Iran. Tel: +98-2188044040, Fax: +98-2188058912, E-mail: Abdialya@ \\ alzahra.ac.ir \\ Received: October 28, 2012; Revised: January 12, 2013; Accepted: January 23, 2013
}

\begin{abstract}
Background: Biofilms are communities of bacteria attached to the surfaces in an extracellular polymeric matrix which are associated with many chronic infections in humans. Acinetobacter spp. are emerging as a major cause of nosocomial infections and Acinetobacter baumannii is the predominant species associated with this kind of infections.

Objectives: In the present study, the potential of biofilm formation of clinical isolates, A. baumannii, was assessed by using crystal violet method. Furthermore, susceptibility pattern of these strains to ciprofloxacin and imipenem was determined.

Methods and Materials: Biofilm formation by 75 A. baumannii isolates was evaluated by using microtiter plate and tube methods and crystal violetstaining. Tube method was carried out under static and shaking conditions. Then, the susceptibility of isolates to ciprofloxacin and imipenem was determined.

Results: Results showed that in tube method under shaking, $22 \%$ of clinical isolates were strong biofilm producers while $23 \%$ of them were not able to form biofilms. In this experiment, $18 \%$ and $42 \%$ of isolates were considered as moderate and weak biofilm-forming strains, respectively. In microtiter plate tests, $18 \%$ of strains were strong-biofilm producers and $25 \%$ of them were notable biofilm producers. In this assessment, $10 \%$ and $47 \%$ were considered as moderate and weak biofilm-forming isolates, respectively. The susceptibility tests, using microdilution method, confirmed that $92 \%$ of these isolates were resistant and $6.6 \%$ were susceptible to ciprofloxacin, although these results for imipenem were $68 \%$ and $24 \%$, respectively.

Conclusions: It can be concluded that most of A. baumannii isolates can form biofilm in microtiter plate and tube. The results also verified that most of these isolates were resistant to ciprofloxacin and imipenem.
\end{abstract}

Keywords: Acinetobacter baumannii; Biofilm; Susceptibility Test; Ciprofloxacin; Imipenem

\section{Background}

Biofilms are highly structured communities of bacteria enclosed to the surfaces that are attached in an extracellular polymeric matrix, exhibiting a modified phenotype compared with corresponding planktonic cells, especially in gene transcription, as well interaction with each other. This structure is identified as a common cause of human infection (1-4). Bacterial biofilms have been found on the surface of different instruments such as intubation tubes, catheters and artificial heart valves in addition of water pipe lines and cleaning instruments (2). The surfaces are usual targets of complex microbial communities (5).

Acinetobacter spp. are ubiquitous, non-fermentative Gram negative bacteria, able to colonize in patients in intensive care units. Acinetobacter baumannii is the main species related to outbreaks of nosocomial infections. This microorganism is an important opportunistic noso- comial pathogen causing epidemic pneumonia, urinary tract infections, septicemia and meningitis (6). Epidemic strains of $A$. baumannii are noted for both intrinsic resistance to antibiotics and their abilities to acquire genes, encoding resistance determinants. Main mechanisms of resistance to $\beta$-Lactams and aminoglycosides are through the production of $\beta$-lactamases and aminoglycosidemodifying enzymes. Moreover, diminished expression of outer membrane proteins, mutations in topoisomerase and up-regulation of efflux pumps play important roles in antibiotic resistance (7).

According to the epidemiologic studies, Acinetobacter biofilms play a role in infectious diseases such as cystic fibrosis, periodontitis, bloodstream infection and urinary tracts infection because of their ability to indwell medical devices (8).

Carbapenems are the preferred treatment for severe Acinetobacter infections, and have reserved better potential

Implication for health policy/practice/research/medical education:

Clinical isolates of A. baumanii have the ability to form biofilms which often cause nosocomial infections and chronic illnesses. Since bacterial popoulations in biofilm are usually more resistant to antibiotics, determination of the relationship between the potential to form biofilm and antibiotic resistance, may be helpful in choosing suitable antibiotic therapy. The multidrug-resistant A. baumannii is a serious concern of health professionals and often leaves few therapeutic options. This survey determines the resistance of $A$. baumannii to common antibiotics.

Copyright (C) 2014 Ahvaz Jundishapur University of Medical Sciences; Published by Kowsar Corp. This is an open-access article distributed under the terms of the Creative Commons Attribution License, which permits unrestricted use, distribution, and reproduction in any medium, provided the original work is properly cited. 
effect than other antimicrobial agents. During the last few years, carbapenem-resistant Acinetobacter isolates have been reported all over the world (9). In 2001, the international network for the study and prevention of emerging antimicrobial resistance (INSPEAR) define the emergence of carbapenem resistance in Acinetobacter as a 'global sentinel event', warranting prompt epidemiological and microbiological interventions (8).

Over the last decades, a broad range of models have been explained for the in vitro study of biofilm formation and development (10). These can be grouped into biofilm biomass assays (based on the quantification of matrix and both living and dead cells), viability assays (based on the quantification of viable cells) and matrix quantification assays (based on the specific staining of matrix components).

\section{Objectives}

In the present study, the potential of biofilm formation by 75 clinical $A$. baumannii strains, isolated from burn wounds and urinary catheters was evaluated by using microtiter plate and tube methods and crystal violet staining, and the susceptibility of the isolates to ciprofloxacin and imipenem was determined.

\section{Materials and Methods}

\subsection{Bacterial Strains and Cultural Conditions}

A total of 75 A. baumannii isolates were collected from different clinical sources, including burn wounds (from burn hospital) and urinary catheters (from an army hospital) and evaluated. Frozen stocks were prepared in Skim milk (Merck, Germany) containing 15\% glycerol (Merck, Germany) and were stored at $-70{ }^{\circ} \mathrm{C}$. All isolates were transferred from the stock cultures into Tryptic soy agar (TSA) (Merck, Germany) and were aerobically incubated at $37^{\circ} \mathrm{C}$ for 24 hours. Pseudomonas aeroginosa PA01 was used as positive control for biofilm formation tests and P. aeroginosa ATCC 27853 served as quality control for susceptibility tests.

\subsection{Biofilm Formation}

Cultures were inoculated in Tryptic soy broth (TSB) (Merck, Germany) and adjusted to 0.5 McFarland standards. Each three wells of a non-adherence, sterile 96-well flat-bottomed were filled with $200 \mu \mathrm{L}$ of bacterial suspension. Negative controls contained only TSB. Then, plates were covered and aerobically incubated for 24 hours at $37^{\circ} \mathrm{C}$. Afterward, the content of each well was aspirated, rinsed five times with $250 \mu \mathrm{L}$ of sterile physiological saline, emptied and left to dry. Then, the plates were stained for 5 minutes with $0.2 \mathrm{~mL}$ of $2 \%$ crystal violet (Merck, Germany). The excess of the stain was rinsed off by insertion the plate under running tap water. Later the plates were air dried; the dye bound to the adherent cells was resolubilized with $160 \mu \mathrm{L}$ of 33\% (v/v) glacial acetic acid. By using an ELISA reader, the OD of each well measured at $650 \mathrm{~nm}$ $(10,11)$.

Biofilm was also formed in test tubes. For this reason, $0.1 \mathrm{~mL}$ of bacterial culture obtained as above mentioned, was transferred to glass test tubes containing $10 \mathrm{~mL}$ TSB. The cultures, under two different conditions: agitation at $200 \mathrm{rpm}$. and stationary were incubated at $37^{\circ} \mathrm{C}$ for 72 hours, following which the medium was removed and tubes were washed with distilled water, air dried and biofilm formation were assayed by crystal violet (7). All tests were carried out in triplicates.

\subsection{Statistical Analysis}

SPSS 16 and one way ANOVA were used to calculate the differences between microtiter plate and tube methods under shaking and static conditions. Experiment was performed in triplicate. P values of $\leq 0.05$ were considered as significant.

Table 1. Percent of A. baumannii Isolates Formed Biofilm in Test Tubes and Microtiter Plates

\begin{tabular}{lllll}
\hline Test & \multicolumn{3}{c}{ Isolates, \% } \\
\cline { 2 - 5 } & Weak Biofilm & Moderate Biofilm & Strong Biofilm & No Biofilm Producer \\
\hline Test tubes & 42 & 18 & 22 & 18 \\
\hline Shaking condition & 42 & 16 & 21 & 23 \\
\hline Static condition & 40 & 10 & 18 & 25 \\
\hline Microtiter plates & 41 & &
\end{tabular}

\subsection{Susceptibility Testing}

All tests were carried out in duplicates. Minimum inhibitory concentrations (MICs) were carried out according to the clinical laboratory standards institute (CLSI) guidelines by using microtiter plate method. To this rea- son, colonies from TSA were suspended in Muller Hinton broth (Merck, Germany) and adjusted to 0.5 McFarland and 1:100 diluted in Muller Hinton broth to reach to a final concentration of $1 \times 10^{6} \mathrm{CFU} / \mathrm{mL}$.

Dilutions of ciprofloxacin and imipenem (Exir, Iran) 
Abdi-Ali A et al.

were made in distilled water and phosphate buffer 0.01 $\mathrm{M}$, respectively. The antibiotics were prepared at different concentrations ranged from 0.25 to $512 \mu \mathrm{g} / \mathrm{mL}$. Each well filled with $100 \mu \mathrm{L}$ of different dilution of the antibiotic and $100 \mu \mathrm{L}$ of bacterial suspension. Microtiter plates were incubated at $37^{\circ} \mathrm{C}$ for 24 hours. MIC was determined as the minimum antibiotic concentration that inhibited the visible growth (12).

\section{Results}

The results determined the rate of adherence of the cells and ability to form biofilm of Acinetobacter isolates. Summarized results of the tube and microtiter-plate tests are presented in Table 1 . Statistical analyses were carried out and the tube test and microtiter-plates showed significantly different results. For a comparative analysis, we used classification of adherence.

The adhesion of isolates is classified into four categories. Strains were classified as follows (10):
a) OD $\leq$ ODc $=$ Non-adherent
b) ODc $\angle$ OD $\leq 2 \mathrm{ODc}=$ Weakly adherent
c) 3 ODc $\angle$ OD $\leq 4 O D c=$ Moderately adherent
D) $4 \mathrm{OD}<\mathrm{ODC}=$ Strongly adherent
e) $\mathrm{ODc}=$ OD of control

Results are shown in Table 1. All of isolates were placed in four different categories: strong, moderate, weak and nonbiofilm producers.

As shown in Figure 1 and according to CLSI protocol, the MIC breakpoint of $\geq 4 \mu \mathrm{g} / \mathrm{mL}$ shows ciprofloxacin resistance and $\leq 1 \mu \mathrm{g} / \mathrm{mL}$ susceptibility to this antibiotic. Consequently, $92 \%$ of isolates were resistant, $6.6 \%$ of isolates were susceptible and $1.4 \%$ of isolates were not susceptible or resistant to ciprofloxacin. As shown in Figure 2, based on CLSI table 2B-2 MICs that are $\leq 4 \mathrm{~g} / \mathrm{mL}$ are susceptible, and $\geq 16 \mathrm{~g} / \mathrm{mL}$ are resistant. Therefore, $68 \%$ of isolates were resistant to imipenem, $24 \%$ were susceptible and $8 \%$ were not susceptible or resistant.

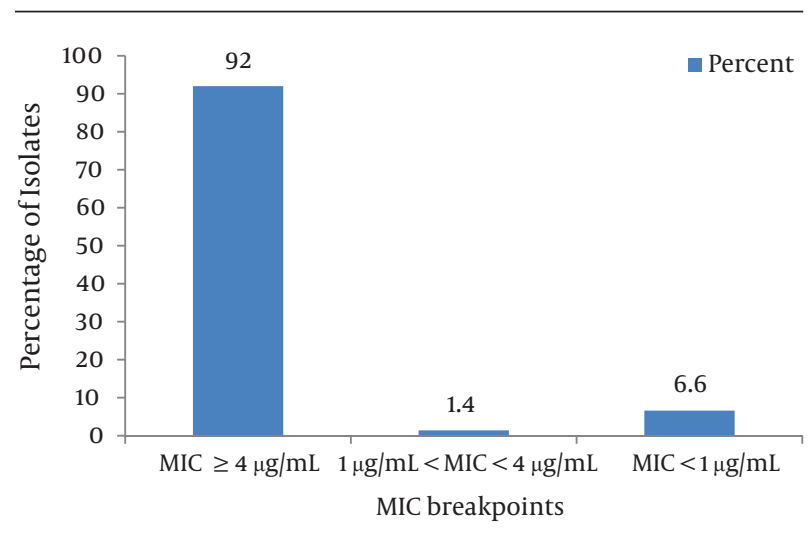

Figure 1. MIC of Ciprofloxacin

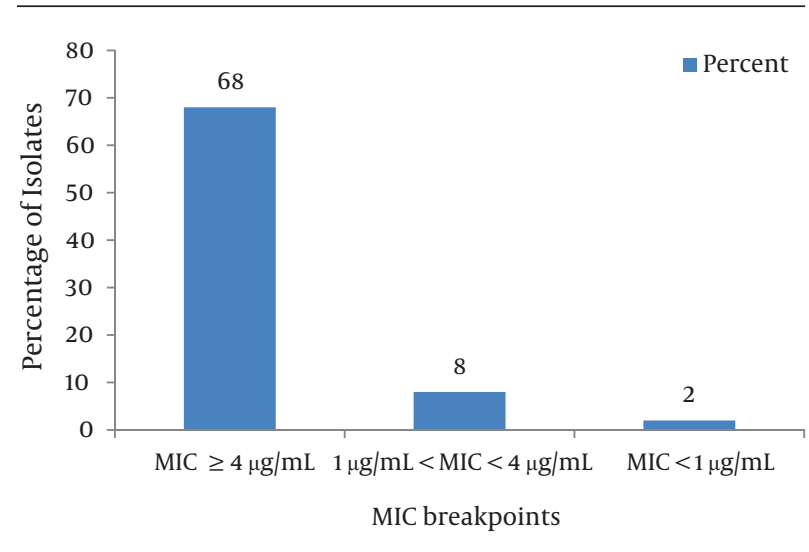

Figure 2. MIC of Imipenem

\section{Discussion}

Biofilms play a major role in microorganism colonization during infection, providing an opportunity for bacteria to develop drug resistance (12). According to the results of the tube tests, approximately all tested strains formed biofilm. The tube adherence assay is not complicated and simple although reading of the results may be complicated. Moreover, observers regularly have different interpretations about weak reactions (10). However, we noted that certain modifications of the process might improve the precision of interpretation of the results obtained by the tube test, e.g. after resolublizing, 200 $\mu \mathrm{L}$ of acetic acid was transferred to a well of a microtiter plate and read by ELISA plate reader, subsequently the approach was changing from a qualitative to a quantitative one.

The quantitative microtiter-plate method predicts clinical applications more reliable than the tube testing (7). In this study, significant disagreement between the tube test results and microtiter-plate was observed. Considerably more strains were classified as weak adherent by the quantitative microtiter-plate test. Factors that may influence the adherence of Acinetobacter are the hydrophobicity of the test tubes and the shaking which increases the chances of microorganisms interaction with the glass surface and uniform dispersion of the nutrients.

Microtiter plate method is a very susceptible, precise, reproducible and affordable method for screening the biofilm formation and can function as a reliable quantitative method for determining biofilm formation. Biofilm formation by A. baumannii might increase the colonization and persistence of bacteria that may lead to higher rates of device related infections. In our microtiter-plate method, the addition of acetic acid permits to measure the attached bacteria both to the bottom and walls of the wells. Only $160 \mu \mathrm{L}$ of $33 \%$ (v/v) glacial acetic acid was added 
per well, to evade interference with stained matter at the liquid-air interface, which was not considered to be indicative of biofilm formation in the tube tests (10).

Resistance patterns amongst nosocomial bacterial pathogens may generally be different from country to country and within a country over time. Because of these differences, a surveillance of nosocomial pathogens resistance is required for each country to show suitable selection for empiric therapy. In addition resistance monitoring could be predict an outbreak. Detection of resistance in a particular pattern may propose a presently occurring epidemic in the hospital (12).

Antibiotic resistance is the main cause of treatment failure of infected patients with all Acinetobacter species, particularly those with $A$. baumannii $(13,14)$. The first line therapy for Acinetobacter infections are amikacin, imipenem, ceftazidime, or a quinolone. Imipenem monotherapy have also been confirmed to be effective (15). However, many current studies have reported the increasing resistance to imipenem $(16,17)$. Most of the latest reports advocated the combination therapy in the present situation to avoid further resistance to imipenem, the antibiotic once considered as the drug of choice for Acinetobacter infections (15).

This high level of resistance to ciprofloxacin may be clarified by the fact that ciprofloxacin has been extensively used in hospitals. Chang et al. (8) reported the highest activity of quinolones against A. baumannii with $97.8 \%$ susceptibility. Imipenem has high affinity to the PBP2 of Gram-negative bacteria, and it has been reported to downregulte the expression of PBP2 which is associated to reduced susceptibility or resistance to carbapenems (18).

The ability of A. baumannii to construct or form biofilms could cause a high level of antibiotic resistance and survival properties (19). This possibility is supported by a very limited number of publications which offered that a clinical isolate of this bacterium is able to attach and form biofilms on glass surfaces $(20,21)$. A. baumannii isolates are capable to form biofilms might be selected under antibiotic pressure, or conversely, A. baumannii might acquire resistance to multiple drugs from the biofilm communities. In either case, it appears that the high colonization capacity of $A$. baumannii, combined with its resistance to multiple drugs, contributes to the organism survival and further dissemination in the hospital setting (22).

In conclusion, the results of study on the formation of biofilms in microtiter plates and test tubes under agitation and stationary growth conditions as well as statistical analysis indicated that the biofilms are formed most frequently under stationary conditions. Isolates of Acinetobacter from urinary catheters were more susceptible to the antibiotics than wound isolates, but isolates from burn wounds were stronger biofilm former than urinary catheters isolates. Multi-drug resistance to antibiotics in wound isolates is just because of the misuse of these an- tibiotics. Consequently, there is a direct relation between increased biofilm formation and antibiotic resistance.

\section{Acknowledgements}

We thank Vice Channcellor of Alzahra University for the financial support.

\section{Authors' Contribution}

None declared.

\section{Financial Disclosure}

None declared.

\section{Funding/Support}

This survey was supported by vice chancellor of Alzahra University, Tehran, Iran.

\section{References}

1. Costerton JW, Stewart PS, Greenberg EP. Bacterial biofilms: a common cause of persistent infections. Science. 1999;284(5418):131822.

2. Donlan RM, Costerton JW. Biofilms: survival mechanisms of clinically relevant microorganisms. Clin Microbiol Rev. 2002;15(2):16793.

3. Lindsay D, von Holy A. Bacterial biofilms within the clinical setting: what healthcare professionals should know. J Hosp Infect. 2006;64(4):313-25.

4. Stoodley P, Sauer K, Davies DG, Costerton JW. Biofilms as complex differentiated communities. Annu Rev Microbiol. 2002;56:187209

5. Heydorn A, Nielsen AT, Hentzer M, Sternberg C, Givskov M, Ersboll BK, et al. Quantification of biofilm structures by the novel computer program COMSTAT. Microbiology. 2000;146 ( Pt 10):2395-407.

6. Van Looveren M, Goossens H, Arpac Steering Group . Antimicrobial resistance of Acinetobacter spp. in Europe. Clin Microbiol Infect. 2004;10(8):684-704.

7. Kazemi Pour Nadia, Dusane Devendra H, Dhakephalkar Prashant K, Zamin Farokh Rokhbakhsh, Zinjarde Smita S, Chopade Balu A. Biofilm formation by Acinetobacter baumannii strains isolated from urinary tract infection and urinary catheters. FEMS Immunol Med Microbiol. 2011;62(3):328-338.

8. Chang Shan-Chwen, Chen Yee-Chun, Luh Kwen-Tay, Hsieh WeiChuan. In vitro activites of antimicrobial agents, alone and in combination, against Acinetobacter baumannii isolated from blood. Diagnos Microbiol Infect Dis. 1995;23(3):105-110.

9. Chu YW, Afzal-Shah M, Houang ET, Palepou MI, Lyon DJ, Woodford $\mathrm{N}$, et al. IMP-4, a novel metallo-beta-lactamase from nosocomial Acinetobacter spp. collected in Hong Kong between 1994 and 1998. Antimicrob Agents Chemother. 2001;45(3):710-4.

10. Stepanović Srdjan, Vuković Dragana, Dakić Ivana, Savić Branislava, Švabić-Vlahović Milena. A modified microtiter-plate test for quantification of staphylococcal biofilm formation. J Microbiological Methods. 2000;40(2):175-179.

11. Ali Oau. Prevention of Proteus mirabilis biofilm by surfactant solution. Egypt Acad J Biolog Sci. 2012;4:1-8.

12. Wikler Matthew A. Performance standards for antimicrobial susceptibility testing: Sixteenth informational supplement.: Clinical and Laboratory Standards Institute; 2006.

13. Gehrlein M, Leying H, Cullmann W, Wendt S, Opferkuch W. Imipenem resistance in Acinetobacter baumanii is due to altered penicillin-binding proteins. Chemotherapy. 1991;37(6):405-12.

14. Rao RS, Karthika RU, Singh SP, Shashikala P, Kanungo R, Jayachandran $S$, et al. Correlation between biofilm production and multiple drug resistance in imipenem resistant clinical isolates of Aci- 
netobacter baumannii. Indian J Med Microbiol. 2008;26(4):333-7.

15. Deighton MA, Balkau B. Adherence measured by microtiter assay as a virulence marker for Staphylococcus epidermidis infections. J Clin Microbiol. 1990;28(11):2442-7.

16. Beck-Sague CM, Jarvis WR, Brook JH, Culver DH, Potts A, Gay E, et al. Epidemic bacteremia due to Acinetobacter baumannii in five intensive care units. Am J Epidemiol. 1990;132(4):723-33.

17. Vidal R, Dominguez M, Urrutia H, Bello H, Garcia A, Gonzalez G, et al. Effect of imipenem and sulbactam on sessile cells of Acinetobacter baumannii growing in biofilm. Microbios. 1997;91(367):7987.

18. Seifert H, Baginski R, Schulze A, Pulverer G. Antimicrobial susceptibility of Acinetobacter species. Antimicrob Agents Chemother. 1993;37(4):750-3.

19. Vidal R, Dominguez M, Urrutia H, Bello H, Gonzalez G, Garcia A, et al. Biofilm formation by Acinetobacter baumannii. Microbios. 1996;86(346):49-58

20. Bauernfeind A, Kljucar S, Jungwrith R. Overview of antibiotic resistance problems in Acinetobacter spp. In: Clinical importance and antibiotic resistance of Acinetobacter spp. J Med Microbiol. 1997(46):726-8.

21. Tolker-Nielsen T, Brinch UC, Ragas PC, Andersen JB, Jacobsen CS, Molin S. Development and dynamics of Pseudomonas sp. biofilms. J Bacteriol. 2000;182(22):6482-9.

22. Fernandez-Cuenca F, Martinez-Martinez L, Conejo MC, Ayala JA, Perea EJ, Pascual A. Relationship between beta-lactamase production, outer membrane protein and penicillin-binding protein profiles on the activity of carbapenems against clinical isolates of Acinetobacter baumannii. J Antimicrob Chemother. 2003;51(3):565-74. 